\title{
Cardiac Tamponade as a Very Rare Complication of Pseudoaneurysm of Mitral Aortic Intervalvular Fibrosa: A Case Report
}

\author{
Fereshteh Ghaderi, Assistant Prof, ${ }^{1}$ Hamid Hosseinikhah, Assistant Prof, ${ }^{2}$ Pouya Nezafati, MD ${ }^{3,4}$
}

${ }^{1}$ Fellowship of Echocardiography, Atherosclerosis Prevention Research Center, School of medicine, Mashhad University of Medical Sciences, Mashhad; ${ }^{2}$ Department of Cardiac Surgery, Imam Reza Hospital, Mashhad University of Medical Sciences, Mashhad; ${ }^{3}$ Department of Cardiac Surgery, Ghaem Hospital, Mashhad University of Medical Sciences, Mashhad; ${ }^{4}$ Cardiac Rehabilitation Research Center, Cardiovascular Research Institute, Isfahan University of Medical Sciences, Isfahan, Iran

\section{ABSTRACT}

Introduction: The mitral-aortic intervalvular fibrosa (MAIVF) has a vital role in the integrity of the mitral and aortic valves. Pseudoaneurysm of the MAIVF (P-MAIVF) usually ensues as a complication of aortic valve surgery, and endocarditis can itself result in several catastrophic complications.

Case Presentation: A 54-year-old male with acute dyspnea was admitted to our hospital. Upon physical and cardiac examination, low blood pressure, low $\mathrm{O} 2$ saturation, tachycardia, and a muffled heart were observed. Findings from the transthoracic echocardiography (TTE) were normal besides a massive pericardial effusion with clot formation in the pericardial space, and a pulsatile echo-free space at the posterior aspect of the aortic prosthesis. Accordingly, transesophageal echocardiography (TEE) was performed and confirmed the presence of a P-MAIVF that was rupturing into the pericardial space, causing cardiac tamponade. The patient presented with an unstable hemodynamic situation. A cardiac surgical operation was planned, however, cardiac arrest occurred at the time of induction. The operation was unsuccessful, and the patient expired in the operating room.

Conclusions: Awareness of P-MAIVF formation after cardiac surgery, particularly after aortic valve replacement (AVR) due to an infected bicuspid aortic valve, is vital in order to prevent the occurrence of cardiac tamponade, a fatal event following this phenomenon. TEE is a modality of choice for identifying P-MAIVF in selected patients.

\section{INTRODUCTION}

The mitral-aortic intervalvular fibrosa (MAIVF) is the region of fibrous tissue between the left half of non-coronary cusp, the adjacent third of left coronary cusp of the aortic valve, and the anterior mitral leaflet. It is of great functional and clinical importance due to its location and its role in the

Received Fuly 25, 2017; accepted April 27, 2018.

Correspondence: Pouya Nezafati, MD, Cardiac Rehabilitation Research Center, Cardiovascular Research Institute, Isfahan University of Medical Sciences, Isfahan, Iran;+989151578421. Fax: +985136078099 (email:pouya.nezafati@gmail.com) functional integrity of mitral and aortic valves [Xie 2013; Rodrigues-Borges 2002]. The MAIVF is the weakest portion of the aortic ring, due to its avascular and fibrous entity [Xie 2013; Kassim 2010; Rodrigues-Borges 2002].

Pseudoaneurysm in the region of the fibrous body between the mitral and aortic valve (AV)- the MAIVF- is a rare but a potentially catastrophic complication. Infective endocarditis and aortic valve surgery are the most frequently associated causative factors. The MAIVF region is a relatively avascular area, and is prone to infection and subsequent aneurysm formation. The formation of a fistulous tract, coronary artery compression, rupture into the pericardium, and sudden death are serious complications that warrant surgical considerations. Herein, we report a rare complication of the MAIVF in a case with an acute dyspnea.

\section{CASE PRESENTATION}

A 54-year-old male patient with a history of aortic valve replacement (AVR) due to an infected bicuspid aortic valve

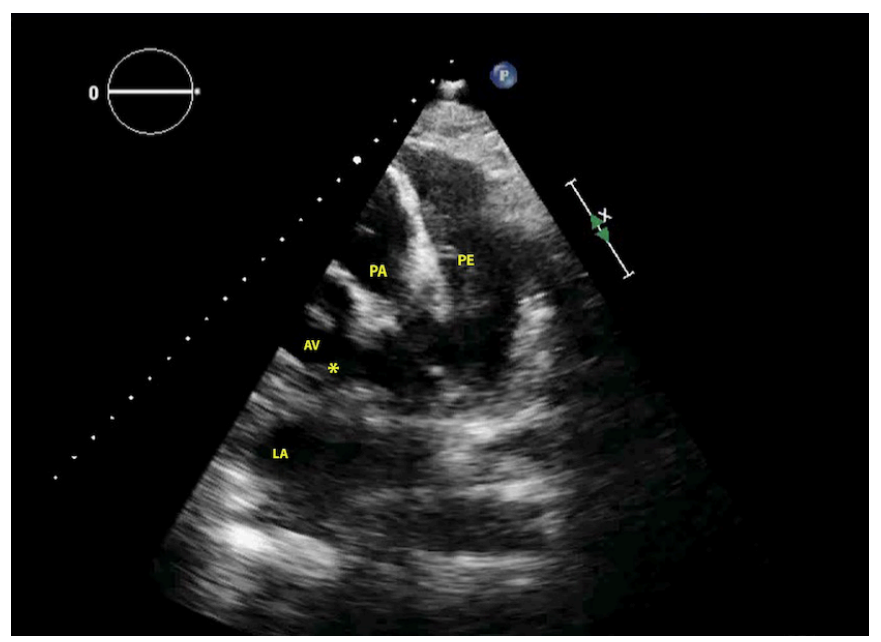

Figure 1. Transthoracic echocardiography in short-axis view showing massive pseudoaneurysm, and the echo free space located at the posterior aspect of the aortic valve prosthesis. PE indicates pericardial effusion; PA, pulmonary artery; P-MAIVF, Pseudoaneurysm of mitral-aortic Intervalvular fibrosa; $\mathrm{AV}$, aortic valve. 


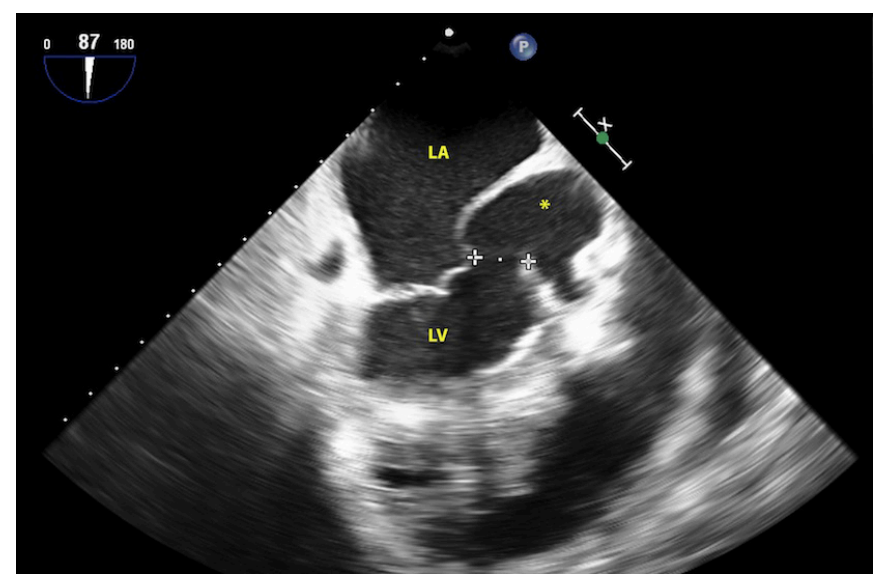

Figure 2. Transesophageal long-axis view showing pseudoaneurysm of the mitral-aortic intervalvular fibrosa (asterisk) communicate with LV through a wide neck ( $14.7 \mathrm{~mm}$, indicated by pluses). AV indicates aortic valve; LA, left atrium; LV, left ventricle.

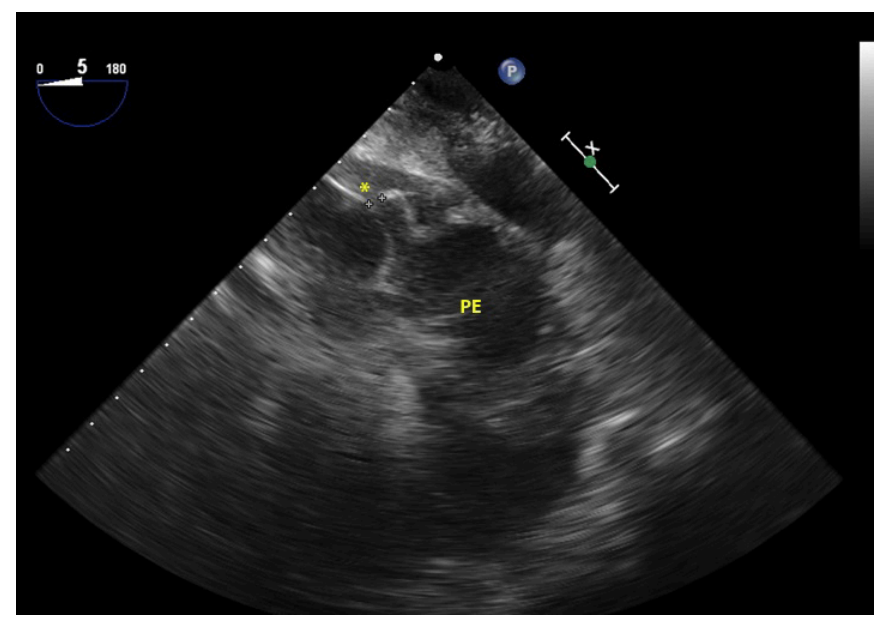

Figure 3. Transesophageal echocardiogram showing pseudoaneurysm of the mitral-aortic intervalvular fibrosa (asterisk) communicating with pericardial sac via a narrow neck ( $4 \mathrm{~mm}$ ) (indicated by pluses), resulting in massive consolidated pericardial effusion (PE).

was admitted to the hospital with acute dyspnea (New York Heart Association functional class IV). He had undergone AVR with a mechanical valve (St. Jude Medical, Inc., USA) six months prior to presentation.

Upon admission, the patient's hemodynamic condition was evaluated through a physical examination, and his vital signs were assessed. His blood pressure, heart rate, and $\mathrm{O} 2$ saturation were $75 / 45 \mathrm{mmHg}, 125 \mathrm{bpm}$, and $86 \%$, respectively, and no fever was detected. During his cardiac examination, a muffled heartbeat with no audible murmur on auscultation was heard. The electrocardiogram revealed a low voltage and sinus tachycardia. He was then transferred to the coronary care unit, and transthoracic echocardiography (TTE) was performed, revealing massive pericardial effusion with clot formation in the pericardial space, and a pulsatile echo-free space at the posterior aspect of the aortic prosthesis (Figure

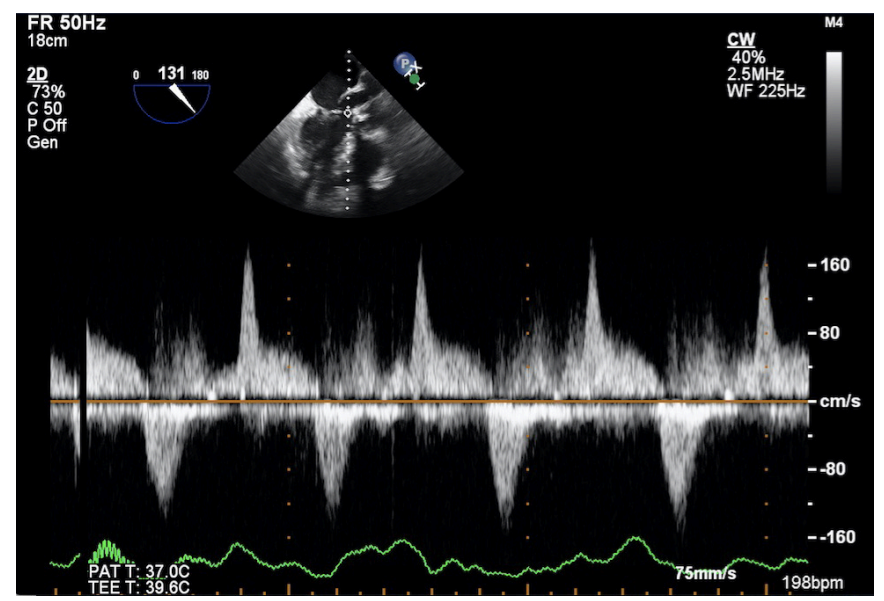

Figure 4. Doppler echocardiography visualizing blood flow in and out of the pseudoaneurysm of the left ventricular outflow tract.

1). The TTE showed normal biventricular systolic function, and a normally functioning aortic valve (AV) with an acceptable transvalvular gradient for the patient's specific type of metallic AV prosthesis.

Transesophageal echocardiography (TEE) was performed to further describe the details of the structure prior to surgery. The TEE showed a large MAIVF pseudoaneurysm measuring approximately $3.7 \mathrm{~cm}$ at its largest dimension. The pseudoaneurysm was located at the posterior aspect of the aortic root, with a wide neck $(13 \mathrm{~mm})$ through the left ventricular outflow tract (LVOT), and rupturing via a narrow neck $(3 \mathrm{~mm})$ into the pericardial space (Figures 2 and 3 ).

Color Doppler echocardiography showed blood flow into the pseudoaneurysm in the systole and outward flow in the diastole, indicating significant communication with the LVOT (Figure 4). There was a similar flow pattern into the pericardial space adjacent to the pulmonary artery, demonstrating a narrow connection site $(3 \mathrm{~mm})$ between the pseudoaneurysm and the pericardial sac (Figure 4).

An urgent cardiothoracic surgery was planned; however, cardiac arrest occurred at the time of induction. As a result, a sternotomy was performed immediately, with a plan for initial stabilization using volume resuscitation and vasopressor infusion. All findings of the echocardiogram were confirmed during surgery. The pseudoaneurysm's site of origin was the aorto-mitral curtain, below the junction of the left and the non-coronary cusps of the aortic annulus. In addition, the eventual pseudoaneurysm that leaked into the pericardium extended from this site, through the roof of the left atrium, and out through the left atrial wall into the pericardial space. Despite the corrective surgery performed, the patient could not survive because of his unstable hemodynamic condition and circulatory collapse.

\section{DISCUSSION}

The thinness and fragility of the region of the fibrous body between the mitral and AV increases its susceptibility to infection and dilatation following endocarditis, mitral and aortic 
valve surgeries, or thoracic trauma. This susceptibility leads to dehiscence in the MAIVF and communication with the LVOT, creating a pseudoaneurysm of the MAIVF (P-MAIVF) [Lin 2015]. Moreover, other cardiac surgical or interventional procedures, including ventricular septal defect repair, cardiac catheterization, radio-frequency catheter ablation of atrial fibrillation, and mitral valve surgery with a maze procedure, may cause injury to the region of the MAIVF, resulting in P-MAIVF. In addition, patients with bicuspid aortic valves are more prone to P-MAIVF, probably because of their congenital weakness in the MAIVF area [Kahveci 2008].

Among the aforementioned risk factors for the formation of P-MAIVF, our patient's history of a bicuspid aortic valve and mechanical AVR could have caused his condition. The P-MAIVF communicates directly with the LVOT and is bounded posteriorly by the left atrium, anteriorly by the aortic root and pulmonary artery, and superiorly by the pericardium [Xie 2013; Sudhakar 2010]. Studies have described surgical methods that can be used for the reconstruction of a P-MAIVF, and the resulting prevention of its fatal complications [Lima 2016; David 1995].

Coronary artery compression and fistula formation are the two most common complications of P-MAIVF [Bonou 2015]. Compression of the coronary artery results in anginal-like symptoms and myocardial infarction; moreover, due to its anatomic proximity, the left main coronary artery is most commonly affected by compression [Bonou 2015; Kassim 2010]. Compression of the pulmonary artery with resultant pulmonary hypertension and compression of the anterior mitral leaflet leading to mitral regurgitation have also been reported [Caselli 2011; Harish 2009]. Fistula formation with adjacent structures, including the left atrium (LA) and aorta, results in heart failure symptoms. In addition, the embolization of thrombotic material in the P-MAIVF may result in a cerebrovascular accident and systemic embolism [Kassim 2010]. Rarely, the P-MAIVF can rupture toward the anterior chest wall, leading to a chest wall mass. An uncommon complication of P-MAIVF rupture into the pericardial space is pericardial tamponade, as occurred in our patient [Xie 2013; Agirbasil 1999]. We believe it would have been highly beneficial if prompt consideration had been given to putting our patient on cardiopulmonary bypass prior to opening the chest, or at least prior to opening the pericardium.

Because of its posterior location, a P-MAIVF may not be visualized clearly with TTE, and may not even be readily identified at surgery. Thus, the formation of P-MAIVF due to our patient's infected bicuspid aortic valve, as the indication for his previous AVR, could have been present and neither identified in his pre-surgical TTE imaging nor detected at his surgery for AVR [Afridi 1995].

The diagnostic sensitivity of TTE for detecting P-MAIVF is $43 \%$, whereas that of TEE is $90 \%$ [Afridi 1995]. Afridi et al produced the largest published series of case studies involving pseudoaneurysms of the MAIVF, and compared the sensitivity of TTE and TEE in detecting them with respect to intraoperative findings. Among 14 patients with pseudoaneurysms of the MAIVF, TTE could diagnose only 6 (43\%), while TEE detected all cases [Xie 2013; Afridi 1995].

The most distinct echocardiographic feature of P-MAIVF is an echo-free space between the posterior portion of the aortic root and the LA, with marked pulsatility, systolic expansion, and diastolic collapse. This distinguishes it from an aortic ring abscess, which does not display this phenomenon [Sadat 2012].

Rupture of a P-MAIVF can be detected by color Doppler, which shows a pseudoaneurysm communicating with the LA, aorta, or even pericardial space [Kassim 2010]. When a fistula with the LA is created, color Doppler echocardiography shows an eccentric jet of regurgitation, mimicking supraannular mitral regurgitation [Xie 2013]. TEE will confirm the origin of the eccentric jet. When a fistula is formed with the aorta, it results in aortic insufficiency, and the pulsatility of the pseudoaneurysm is less pronounced in comparison with that of an unruptured pseudoaneurysm. Rupture into the pericardial space is the most feared complication, but is very rare [Xie 2013; Ekici 2014; Caselli 2011].

\section{CONCLUSION}

Rupture of a P-MAIVF into the pericardial cavity can result in cardiac tamponade, and its catastrophic complications. We strongly suggest that patients with a history of aortic valve surgery, especially due to an infected bicuspid valve, be examined periodically by TTE. If necessary, TEE should be used as an ideal imaging technique for the rapid detection of P-MAIVF. We also suggest that these patients undergo appropriate surgical corrections for the prevention of life-threatening complications from this condition.

\section{REFERENCES}

Afridi I, Apostolidou MA, Saad RM, et al. 1995. Pseudoaneurysms of the mitral-aortic intervalvular fibrosa: dynamic characterization using transesophageal echocardiographic and Doppler techniques. J Am Coll Cardiol 25:137-45.

Agirbasli M, Fadel B. 1999. Mpseudoaneurysm of the Mitral-Aortic Intervalvular Fibrosa: A Long-Term Complication of Infective Endocarditis.Echocardiography 16:253-57.

Bonou M, Papadimitraki ED, Vaina S, et al. 2015. Mitral-Aortic Intervalvular Fibrosa Pseudoaneurysm. J Cardiovasc Ultrasound 23:257-61.

Caselli S, Mazzesi G, Tritapepe L, et al. 2011. 3D echocardiographic delineation of mitral-aortic intervalular fibrosa pseudoaneurysm caused by bicuspid aortic valve endocarditis. Echocardiography 28:E1-4.

David TE, Feindel CM, Armstrong S, et al. 1995. Reconstruction of the mitral annulus: A 10 year experience. J Thorac Cardiovasc Surg 110:1323-32.

Ekici F, Kocabaş A, Aktaş D, et al. 2014. Native aortic valve endocarditis complicated by pseudoaneurysm of mitral-aortic intervalvular fibrosa. Echocardiography 31:E60-3.

Harish A, Hatibu S, Ramkumar SR, et al. 2009. Aneurysm of mitral aortic intervalvular fibrosa- a case report and review. Indian Heart J 61:394-6.

Kahveci G, Yilmaz F, Mutlu B. 2008. Bicuspid aortic valve endocarditis complicated with aortic valve aneurysm and pseudoaneurysm of the mitral-aortic intervalvular fibrosa. Echocardiography 25:799-801. 
Kassim TA, Lowery RC, Nasur A, et al. 2010. Pseudoaneurysm of mitralaortic intervalvular fibrosa: two case reports and review of literature. Eur J Echocardiogr 11:E7.

Lima B, Chamogeorgakis T, MacHannaford JC, et al. 2016. How to do it: The commando operation for reconstruction of the fibrous skeleton with double valve replacement. Heart Surg Forum 19:E308-10

Lin A, Poppas A, Mansoor A, et al. 2015. Pseudoaneurysm of the mitralaortic intervalvular fibrosa. J Cardiovasc Med (Hagerstown) $16 \mathrm{Suppl}$ 1:S33-4.

Rodrigues-Borges AG, Suresh K, Mirza H, et al. 2002. False aneurysm of the mitral-aortic intervalvular fibrosa after uncomplicated aortic valve replacement. J Am Soc Echocardiogr 15:743-5.

Sadat K, Joshi D, Sudhakar S, et al. 2012. Incremental role of threedimensional transesophageal echocardiography in the assessment of mitral-aortic intervalvular fibrosa abscess. Echocardiography 29:742.

Sudhakar S, Sewani A, Agrawal M, et al. 2010. Pseudoaneurysm of the mitral-aortic intervalvular fibrosa (MAIVF): A comprehensive review. J Am Soc Echocardiogr 23:1009-18.

Xie M, Li Y, Cheng TO, et al. 2013. Pseudoaneurysm of the mitral-aortic intervalvular fibrosa. Int J Cardiol 166:2-7. 